\title{
Intraspecific variation in elemental accumulation and its association with salt tolerance in Paspalum vaginatum
}

\author{
David M. Goad (D) , ${ }^{1,2}$ Elizabeth A. Kellogg (D) , I Ivan Baxter (D) , ${ }^{1}$ and Kenneth M. Olsen (D) ${ }^{2, *}$ \\ ${ }^{1}$ Donald Danforth Plant Science Center, St. Louis, MO 63132, USA, and \\ ${ }^{2}$ Department of Biology, Washington University in St. Louis, St. Louis, MO 63130, USA \\ *Corresponding author: Department of Biology, Campus Box 1137, Washington University in St. Louis, 1 Brookings Dr. St. Louis, MO 63130, USA. \\ Email: kolsen@wustl.edu
}

\begin{abstract}
Most plant species, including most crops, perform poorly in salt-affected soils because high sodium levels are cytotoxic and can disrupt the uptake of water and important nutrients. Halophytes are species that have evolved adaptations to overcome these challenges and may be a useful source of knowledge for salt tolerance mechanisms and genes that may be transferable to crop species. The salt content of saline habitats can vary dramatically by location, providing ample opportunity for different populations of halophytic species to adapt to their local salt concentrations; however, the extent of this variation, and the physiology and polymorphisms that drive it, remain poorly understood. Differential accumulation of inorganic elements between genotypes or populations may play an important role in local salinity adaptation. To test this, we investigated the relationships between population structure, tissue ion concentrations, and salt tolerance in 17 "finetextured" genotypes of the halophytic turfgrass seashore paspalum (Paspalum vaginatum Swartz). A high-throughput ionomics pipeline was used to quantify the shoot concentration of 18 inorganic elements across three salinity treatments. We found a significant relationship between population structure and ion accumulation, with strong correlations between principal components derived from genetic and ionomic data. Additionally, genotypes with higher salt tolerance accumulated more $\mathrm{K}$ and Fe and less Ca than less tolerant genotypes. Together these results indicate that differences in ion accumulation between $P$. vaginatum populations may reflect locally adapted salt stress responses.
\end{abstract}

Keywords: Paspalum vaginatum; population structure; halophyte; ionomics; salt tolerance

\section{Introduction}

High salt concentrations represent a particularly harsh environment for most terrestrial plants (Parida and Das 2005). As such, only a relatively few specialist species, called halophytes, have evolved to tolerate them. The challenge that wild plant species face in adapting to saline environments in the wild is also apparent in crop breeding, where the production of salt tolerant varieties in otherwise nontolerant (i.e., glycophytic) crop species has proceeded slowly. This is problematic because $25-30 \%$ of irrigated land is estimated to be salt-affected (Shahid et al. 2018) with an additional 10 million hectares becoming salinized each year (Szabolcs 1989). To develop crops that can produce satisfactory yields in these soils, a better understanding of how halophytes has managed to overcome extreme salt stress may be vital (Cheeseman 2015).

Currently, most of our knowledge of the genetic and physiological underpinnings of salt tolerance comes from crop species and plant model species that are not salt tolerant, with limited studies of halophytes. Furthermore, even studies in halophytes have often ignored intraspecific variation in levels of salt tolerance (Cheeseman 2013; Saslis-Lagoudakis et al. 2014). Some halophytic species grow in a wide range of salt concentrations, from brackish estuaries to beaches where they are regularly exposed undiluted seawater. If variation in salt tolerance is heritable, it could allow populations to adapt to the salt concentrations specific to their local environment. This sort of fine-scale adaptation could be immensely useful for not only incrementally increasing the tolerance of crop varieties but also closely matching them to regional salinity conditions.

Ionomics, the quantification of the total inorganic contents of a plant, is a cost-effective and high-throughput method for studying how plants respond to stress (Baxter et al. 2008). Specifically, it can assess differences in the concentration of each element individually or by incorporating the concentration of multiple elements into an "elemental profile" for each plant. It is particularly useful for investigating intraspecific variation in salt tolerance because plants must deal with high external salt concentrations by controlling the uptake of $\mathrm{Na}$ while maintaining the accumulation of other important elements (Kumari et al. 2015). Sodium accumulation can be beneficial for osmoregulation in high salt environments; however, at high concentrations it is toxic. Additionally, Na can compete for uptake with other important elements such as $\mathrm{Mg}, \mathrm{K}$, and $\mathrm{Ca}$, causing nutrient deficiencies (Yermiyahu et al. 1994; Ali and Yun 2017). By measuring 
differences in the concentrations of these and other elements in tissues between different genotypes, ionomics can provide a window into intraspecific variation in tolerance. For example, increased $\mathrm{Na}$ accumulation due to a polymorphism in a sodium transporter gene is associated with adaptation to saline environments in the glycophyte Arabidopsis thaliana (Baxter et al. 2010), and differences in the accumulation of several different ions have been reported between halophytic and glycophytic Lotus species (Sanchez et al. 2011). Studies examining ionomic variation within a halophytic species, however, remain rare.

The model halophyte seashore paspalum (Paspalum vaginatum Swartz) is an ideal model system to ask questions about intraspecific variation in salt tolerance. As a turfgrass with a dedicated breeding program and reference genome, it has been the focus of several studies which documented variation in salt tolerance between breeding lines (Lee et al. 2004a, 2004b, 2005, 2007, 2008). However, these studies have focused on breeding lines that had been collected for their favorable turf qualities and may inadvertently have excluded less salt tolerant genotypes, thereby reducing the potential to detect the phenotypic variation. Additionally, these studies predate genetic characterizations of the study lines, which subsequently revealed extensive genotypic redundancy and potential mislabeling in publicly available germplasm collections (Eudy et al. 2017; Goad et al. 2021). The lack of genotypic data in earlier studies also prevented comparisons between genetically differentiated subpopulations of the species.

While differences in salt tolerance between genotypes of $P$. vaginatum have been recorded, the mechanisms underlying this variation are not well understood. Unlike some halophytes, P. vaginatum does not seem to secrete salt and therefore must deal with sodium toxicity by either excluding sodium ions at uptake or sequestering them in specialized structures (Chen et al. 2009). Recent work has shown that epidermal leaf papillae may act as Na sinks in P. vaginatum, supporting the hypothesis of Na sequestration (Spiekerman and Devos 2020). Notably, these papillae are poorly developed in the closely related glycophyte Paspalum distichum. Variation in salt tolerance may also be controlled by osmoregulation through differential accumulation of organic osmolytes or inorganic ions. In P. vaginatum both proline and potassium accumulation have been shown to be associated with total biomass at high salt concentrations (Lee et al. 2007, 2008) suggesting that they may be involved in salt tolerance responses.

Paspalum vaginatum falls into two morphotypes, a "finetextured" form which is sought out for turf and a "coarsetextured" form which is not. In a recent study, Goad et al. (2021) showed that the fine-textured plants, including those from breeding populations at the US Department of Agriculture (USDA), are uniformly diploid and are genetically distinct from coarse-type accessions, which consist of interspecific hybrids of varying ploidy. That study also uncovered circumstantial evidence suggesting that the fine-textured and coarse-textured morphotypes may differ in their salt tolerance. Furthermore, the study identified genotypic differences among the fine-textured accessions, although it did not explicitly examine their population structure. Because fine-textured accessions provide the primary germplasm for turf breeding, further investigation of them is warranted.

In this study, we have characterized intraspecific variation in salt tolerance in fine-textured $P$. vaginatum as related to genotypic variation in this economically important morphotype. We assayed the shoot biomass and ionome profiles of 44 accessions representing 17 unique genotypes at multiple salinity treatments and performed population genetics analysis on a dataset that included an additional 7 genotypes to ask the following questions: (1) Does ionomic variation among genotypes correspond to genome-wide genetic differentiation (population structure)? (2) Do independently collected and maintained accessions of identical genotypes exhibit different ionomic profiles, indicating phenotypic plasticity for this trait? (3) Do tissue ion concentrations correlate with salt tolerance (measured as change in biomass between low and high salt treatments) for individual genotypes? (4) If so, to what extent are these correlations attributable to population-level differences?

\section{Methods Plant material}

Plants used in salinity tolerance phenotyping experiments were a subset of the fine-textured P. vaginatum accessions examined by Goad et al. (2021), including wild collections in the southeastern United States (Supplementary Figure S1) and USDA GRIN lines. A total of 44 accessions represented 17 unique genotypes (Supplementary Table S1). An additional seven genotypically unique accessions from Goad et al. (2021) were included in the population genetics analysis but were not phenotyped.

\section{Population genetic analyses}

Raw genotyping-by-sequencing (GBS) genotype calls for the 24 unique fine-textured genotypes from Goad et al. (2021) were filtered by removing sites with MAF $<0.05$ and missing data $>0.1$. Population structure was then assessed by principal component analysis (PCA), performed in PLINK (Purcell et al. 2007), and assignment to genetic populations with ADMIXTURE (Alexander et al. 2009). For comparisons between populations, genotypes that were not unambiguously assigned to a single population in ADMIXTURE were placed into populations based on having $\geq 50 \%$ assignment to that population.

\section{Salt tolerance assays}

Three stolon cuttings of each accession, consisting of a single node and connected shoot tissue, were transplanted to a $6 \times 5 \mathrm{~cm}$ pot containing a clay growth medium (Turface Field and Fairway, Profile Products LLC, Buffalo, IL, USA) in the Donald Danforth Plant Science Center Plant Growth Facility. Plants were allowed to establish for 6 weeks and were watered twice daily to runthrough with tap water. For the first week, plants were covered with domes to prevent dehydration. For the next three weeks, they were allowed to grow uncovered. They were then trimmed to $2 \mathrm{~cm}$ above the soil line and allowed to continue growing for the remaining 2 weeks.

Plants were then moved to one of three experimental flood trays where each tray contained one replicate of each accession with its position randomly assigned. A Raspberry Pi computer was programmed to flood all three trays simultaneously with nutrient solution from a shared reservoir twice daily to represent tidal inflows (Supplementary Figure S2). The pump flooding a given tray was automatically shut off once the solution reached a sensor placed $1 \mathrm{~cm}$ above the soil line; the solution then passively drained back into the reservoir over a 30 -min time period. The nutrient solution consisted of $230 \mathrm{~L}$ of a 50/50 mix of tap water and a 2x concentration of Jack's CA-MG 15-5-15 (JR Peters Inc., Allentown, PA, USA) with $\mathrm{NaCl}$ added according to treatment. Plants were allowed to acclimate to the flood tray for 2 days with no added $\mathrm{NaCl}$ before experimental treatments began, at which point they were trimmed to $2 \mathrm{~cm}$ above the soil line. 
Plants in flood trays were exposed to increasing levels of salt over a period of 12 weeks with the salt level increased every 2 weeks after the initial acclimation period, for a total of six experimental treatments (salt levels) for each plant. Increase in the salinity of the nutrient solution was measured by the electrical conductivity of the solution (ECw, units $\mathrm{dS} / \mathrm{m}$ ) at 2-week intervals. The ECw of each treatment and the amount of added $\mathrm{NaCl}$ were as follows: $2.5 \mathrm{dS} / \mathrm{m}=0 \mathrm{~g} \mathrm{~L}^{-1}, 10 \mathrm{dS} / \mathrm{m}=6.9 \mathrm{~g} \mathrm{~L}^{-1}, 20 \mathrm{dS} /$ $\mathrm{m}=13.8 \mathrm{~g} \mathrm{~L}^{-1}, 30 \mathrm{dS} / \mathrm{m}=20.7 \mathrm{~g} \mathrm{~L}^{-1}, 40 \mathrm{dS} / \mathrm{m}=27.6 \mathrm{~g} \mathrm{~L}^{-1}$, and 50 $\mathrm{dS} / \mathrm{m}=34.5 \mathrm{~g} \mathrm{~L}^{-1}$. The first week at each salinity level served as an acclimation period, after which the aboveground tissue of each plant was trimmed to $2 \mathrm{~cm}$ above the soil line and discarded. After the second week at the same salinity level plants were again trimmed to $2 \mathrm{~cm}$; however, this time tissue was saved and dried for at least 1 week before being used in subsequent analyses.

To prevent increases in salinity concentration due to evaporation, the nutrient solution was changed weekly, and electrical conductivity was tested daily with a PINPOINT Salinity Monitor (American Marine, Ridgefield, CT, USA). The conductivity of the solution never exceeded the target value by more than $2.5 \mathrm{dS} / \mathrm{m}$, so no adjustment was necessary over the course of a week. A sensor failure during the acclimation period of the $20 \mathrm{dS} / \mathrm{m}$ treatment resulted in an overflow of one flood tray. The remaining solution was no longer sufficient to fill all three trays so the nutrient solution was replaced with a fresh batch before the next watering event.

\section{Tissue weight measurements and ionomics sample preparation}

Two weights were measured for each dried leaf tissue sample: the total mass of the tissue sample harvested from the plant (collected biomass) and the weight of the subset of that tissue used for ionomics analysis (sample weight). These two values were measured concurrently during ionomics sample preparation. The entirety of each dried sample was transferred to a glass tube and the weight was recorded as the collected biomass. The target weight for the ionomics pipeline was 60-125 mg; therefore, if the collected biomass for a sample was greater than $125 \mathrm{mg}$, tissue was removed from the tube until the sample weight was within the target range. For plants with less than $125 \mathrm{mg}$ collected biomass, the measures for sample weight and collected biomass were identical. Some samples, particularly those in higher salt treatments, had collected biomass weights below the target sample range of $60 \mathrm{mg}$. Ionomics analysis was still performed on these samples, and the effect of low sample weight was controlled statistically as described below.

\section{Ionome measurements}

Concentrations of B, Na, Mg, P, S, K, Ca, Mn, Fe, Co, Ni, Cu, Zn, As, $\mathrm{Se}, \mathrm{Rb}, \mathrm{Mo}$, and $\mathrm{Cd}$ were measured with inductively coupled plasma mass spectrometry (ICP-MS) following the protocol of Ziegler et al. (2013). Briefly, each sample was digested overnight in $2.5 \mathrm{~mL} \mathrm{HNO}_{3}$ containing 20 parts per billion (ppb) indium as an internal standard. Samples were then heated to $100^{\circ} \mathrm{C}$ and diluted to $10 \mathrm{~mL}$ with ultra-pure water containing yttrium as an internal standard. Concentrations for each ion were then measured on a Perkin Elmer Elan 6000 DRC-e mass spectrometer. Reported concentrations were corrected based on the indium and yttrium controls as well as a matrix-matched control containing pooled samples from that run. This matrix match control was repeated after every 10th sample to control for intrarun variation. Because the sample pool differed for each run, we statistically controlled for variation between runs by including the ICP run as an effect in our models as described below.

\section{Data filtering}

Ionomics measurements were performed on samples from all salinity treatments; however, samples from the three highest salt treatments $(30,40$, and $50 \mathrm{dS} / \mathrm{m}$ ) had excessive missing data due to dead plants and low sample weight (7 of 17 genotypes had 1 or fewer samples for the $30 \mathrm{dS} / \mathrm{m}$ treatment; Supplementary Table S2). This meant that sample sizes were inadequate to analyze ion concentration data for the three highest salinity treatments, and they were therefore excluded from subsequent analyses.

For the remaining 346 samples from the 2.5, 10, and $20 \mathrm{dS} / \mathrm{m}$ treatments, we applied a series of data filters. To reduce potential technical variation caused by samples with extremely low weights, we first removed ionomics samples that weighed less than $15 \mathrm{mg}$; this excluded 48 samples. Next, we identified samples with poor-quality data across multiple ion concentrations. To do this, we performed a PCA including the concentration of all ions for every sample. Five outliers were visually identified after plotting PC 1 and PC 2 and subsequently removed. For the remaining samples, we then identified outliers for each ion concentration within each salinity treatment following the algorithm from Davies and Gather (1993) as implemented in the outlierRemoveDataset function in the IonomicsUtils package (https:// github.com/gziegler/ionomicsUtils/, Accessed: 5/30/2019). We used the default parameters which removes outliers surpassing a conservative 6.2 median absolute deviation. This final filtered dataset contained 293 samples (Supplementary Table S3).

\section{Testing for correlations between weight and ion concentration}

To test whether technical variation in elemental concentrations was due to sample weight variation, we performed another PCA on the cleaned dataset and used a linear regression to test for correlations between sample weight and the first two PCs. We also assessed whether variance in PCs could be explained by salinity concentration treatment or flood tray.

Because sample weight and collected biomass were not independent, we then tested whether sample weight correlations could instead be due to actual changes in tissue ion concentration rather than the technical variation due to sample weight. We took the 144 samples that had greater than $60 \mathrm{mg}$ collected biomass and ran a mixed-effects model for each ion concentration using the following formula:

$$
\begin{aligned}
\mathrm{Y}_{\mathrm{ijklm}}= & \mu+\text { treatment }_{\mathrm{i}}+\text { weight }_{j}+\left(\text { treatment } \times \text { weight }_{\mathrm{ij}}+\text { rep }_{k}\right. \\
& +\mathrm{ICP}_{l}+\text { genotype }_{m}+\varepsilon_{i j k l m},
\end{aligned}
$$

where $Y_{i j k l m}$ is the tissue ion concentration; $\mu$ is the overall mean; treatment $t_{i}$ is the ith salinity level; weight $t_{j}$ is the collected biomass of the sample; (treatment $\times$ weight) ij is the effect of the interaction between sample weight and treatment; $\mathrm{rep}_{k}$ is the flood tray as a random effect; $\mathrm{ICP}_{l}$ is the random effect of the lth ICP-MS run; genotype $m$ is the random effect of the $m$ th genotype of the sample; and $\varepsilon_{i j k l m}$ is the random error term.

We then ran the same model except with weight $t_{i}$ as the effect of sample weight rather than collected biomass. If collected biomass was significant at a Bonferroni corrected threshold of $P=2.7 \times 10^{-3}$ and sample weight was not, then the ion concentration was considered to be attributable to actual differences in 
ion accumulation between plants with different collected biomass rather than an artifact of sample weight.

\section{Genotype-by-treatment interactions}

In order to test for significant interactions between genotype and treatment for each ion, we tested a model with the following equation for each ion concentration:

$$
\begin{aligned}
\mathrm{Y}_{\mathrm{ijklm}}= & \mu+\text { treatment }_{i}+\text { genotype }_{j}+(\text { treatment } \times \text { genotype })_{i j} \\
& + \text { weight }_{k}+\operatorname{rep}_{l}+\mathrm{ICP}_{m}+\varepsilon_{i j k l m},
\end{aligned}
$$

where $Y_{i j k l m n}$ is the tissue ion concentration; $\mu$ is the overall mean; treatment $t_{i}$ is the ith salinity level; genotype $e_{j}$ is the random effect of the jth genotype; (treatment $\times$ genotype) ${ }_{i j}$ is the effect of the interaction between genotype and treatment; weight $t_{k}$ is the collected biomass of the sample; rep $_{l}$ is the random effect of the lth flood tray; $\mathrm{ICP}_{m}$ is the random effect of the $m$ th ICP-MS run; and $\varepsilon_{i j k l m}$ is the random error term. We then checked for significance of the (treatment $\times$ genotype $)_{i j}$ term using a Bonferroni corrected threshold of $P=2.7 \times 10^{-3}$.

\section{Within-genotype variation}

To compare the level of variation between both genotypes and accessions within a genotype for each ion concentration, we tested the following nested mixed model:

$$
\begin{aligned}
\mathrm{Y}_{\mathrm{ijklmn}}= & \mu+\text { treatment }_{i}+\text { genotype }_{j}+\left(\text { genotype } \times \text { accession }_{j \mathrm{jk}}\right. \\
& + \text { weight }_{l}+\text { rep }_{m}+\mathrm{ICP}_{n}+\varepsilon_{i j k l m n},
\end{aligned}
$$

where $Y_{i j k l m n}$ is the ion concentration; $\mu$ is the overall mean; treatment $_{i}$ is the ith salinity level; genotype $e_{j}$ is the fixed effect of the jth genotype; (genotype $\times$ accession) jk $_{k}$ is the nested effect of the $k$ th accession within the jth genotype; weight $t_{1}$ is the weight of the ionomics sample; rep $_{m}$ is the random effect of the $m$ th flood tray; $\mathrm{ICP}_{n}$ is the random effect of the $n$th ICP-MS run; and $\varepsilon_{i j k l m n}$ is the random error term. We then checked for significance of the genotype $_{j}$ and (genotype $\times$ accession$_{j k}$ terms using a Bonferroni corrected threshold of $\mathrm{P}=2.7 \times 10^{-3}$.

\section{Genotypic correlations between ion concentration and salt tolerance}

To assay the level of salt tolerance of genotypes, we calculated the percent change in biomass due to increased salinity as the percent change in sampled biomass from the $2.5 \mathrm{dS} / \mathrm{m}$ treatment to the $30 \mathrm{dS} / \mathrm{m}$ treatment using the following equation: (biomass at $30 \mathrm{dS} / \mathrm{m}$-biomass at $2.5 \mathrm{dS} / \mathrm{m}$ )/biomass at $2.5 \mathrm{dS} / \mathrm{m}$. This value was calculated individually for each replicate of a genotype and then averaged. Biomass of samples that had been filtered out of the ionomics analyses due to low sample weight was included in this measure. If a plant had died by the $30 \mathrm{dS} / \mathrm{m}$ treatment, no percent change in biomass was calculated for it and that replicate was not included in the genotypic average. We tested for differences between the three genetic populations identified in our ADMIXTURE analysis for both collected biomass at $2.5 \mathrm{dS} / \mathrm{m}$ and the percent change in biomass using one-way ANOVAs.

For each ion and the K/Na ratio, we then calculated a best linear unbiased prediction (BLUP) for every genotype by extracting the genotype effect for each genotype from the following mixed model:

$$
\begin{aligned}
\mathrm{Y}_{i j k l m}= & \mu+\text { treatment }_{i}+\text { weight }_{j}+\left(\text { treatment } \times \text { weight }_{i j}+\text { rep }_{k}\right. \\
& +\mathrm{ICP}_{l}+\text { genotype }_{m}+\varepsilon_{i j k l m},
\end{aligned}
$$

where $Y_{i j k l m}$ is the adjusted ion concentration; $\mu$ is the overall mean; treatment $t_{i}$ is the ith salinity level; weight is the weight of the ionomics sample; (treatment $\times$ weight) ij $_{\text {ij }}$ is the effect of the interaction between sample weight and treatment; rep $_{k}$ is the flood tray as a random effect; $\mathrm{ICP}_{l}$ is the random effect of the lth ICPMS run; genotype $m$ is the random effect of the $m$ th genotype of the sample; and $\varepsilon_{i j k l m}$ is the random error term.

Since no genotype-by-treatment effects were significant with Equation (2), the interaction term was not included in the model. We then performed a PCA using the genotypic BLUPs as input. To test for genotypic correlations between the percent change in biomass and tissue ion concentration, we performed a Spearman correlation between each BLUP or PC and the average change in biomass as calculated above. Correlations were considered significant with Bonferroni corrected threshold of $\mathrm{P}<2.7 \times 10^{-3}$.

In order to compare differences in elemental accumulation across populations and treatment, we generated another set of BLUPs for elements that were significantly associated with salt tolerance as well as $\mathrm{Na}$ and the K/Na ratio. These BLUPs were calculated independently for each treatment using the following model:

$$
\mathrm{Y}_{\mathrm{ijkl}}=\mu+\text { weight }_{j}+\mathrm{rep}_{j}+\mathrm{ICP}_{k}+\text { genotype }_{l}+\varepsilon_{\mathrm{ijk} k}
$$

where $Y_{i j k l}$ is the adjusted ion concentration; $\mu$ is the overall mean; weight $t_{i}$ is the weight of the ionomics sample; rep $_{k}$ is the flood tray as a random effect; $\mathrm{ICP}_{l}$ is the random effect of the lth ICP-MS run; genotype is the random effect of the lth genotype of the sample; and $\varepsilon_{i j k l}$ is the random error term.

\section{Results \\ Population structure}

Population structure analysis of GBS genotypes revealed three genetic populations in both the PCA and ADMIXTURE analysis (Figure 1). The optimal $\mathrm{K}$ for the ADMIXTURE analysis as determined by the cross-validation (CV) error was 2; however, the CV error for $K=3$ was only slightly higher than $K=2$ and much lower than for all of the other potential $K$ values (Supplementary Figure S3). We chose to use $K=3$ despite this slightly higher CV error because it better reflected groupings in both our genetic and phenotypic PCAs. One genetic population (labeled Population 1) was composed entirely of wild collections from the southeastern US Atlantic Coast and is the most genetically distant from the other two populations, particularly along PC 1 which explained 15.4\% of the variance. PC 2 largely separated population 3 from the other two populations and explained $11.3 \%$ of the variance.

\section{Correlations between sample weight and ion concentration}

To be sure that differences in ion concentration between samples were due to physiological differences and not technical error due to variation in sample weight, we performed a PCA on the samples from the 2.5, 10, and $20 \mathrm{dS} / \mathrm{m}$ treatments. The first two PCs (which explained $27.0 \%$ and $21.8 \%$ of the variance, respectively) both correlated significantly with sample weight (PC 1, $r^{2}=0.14$, $\mathrm{P}<10^{-11}$; PC 2, $r^{2}=0.26, P<10^{-16}$; Supplementary Figure S4A). However, PC 2 was largely driven by salinity treatment $\left(r^{2}=0.62\right.$, 

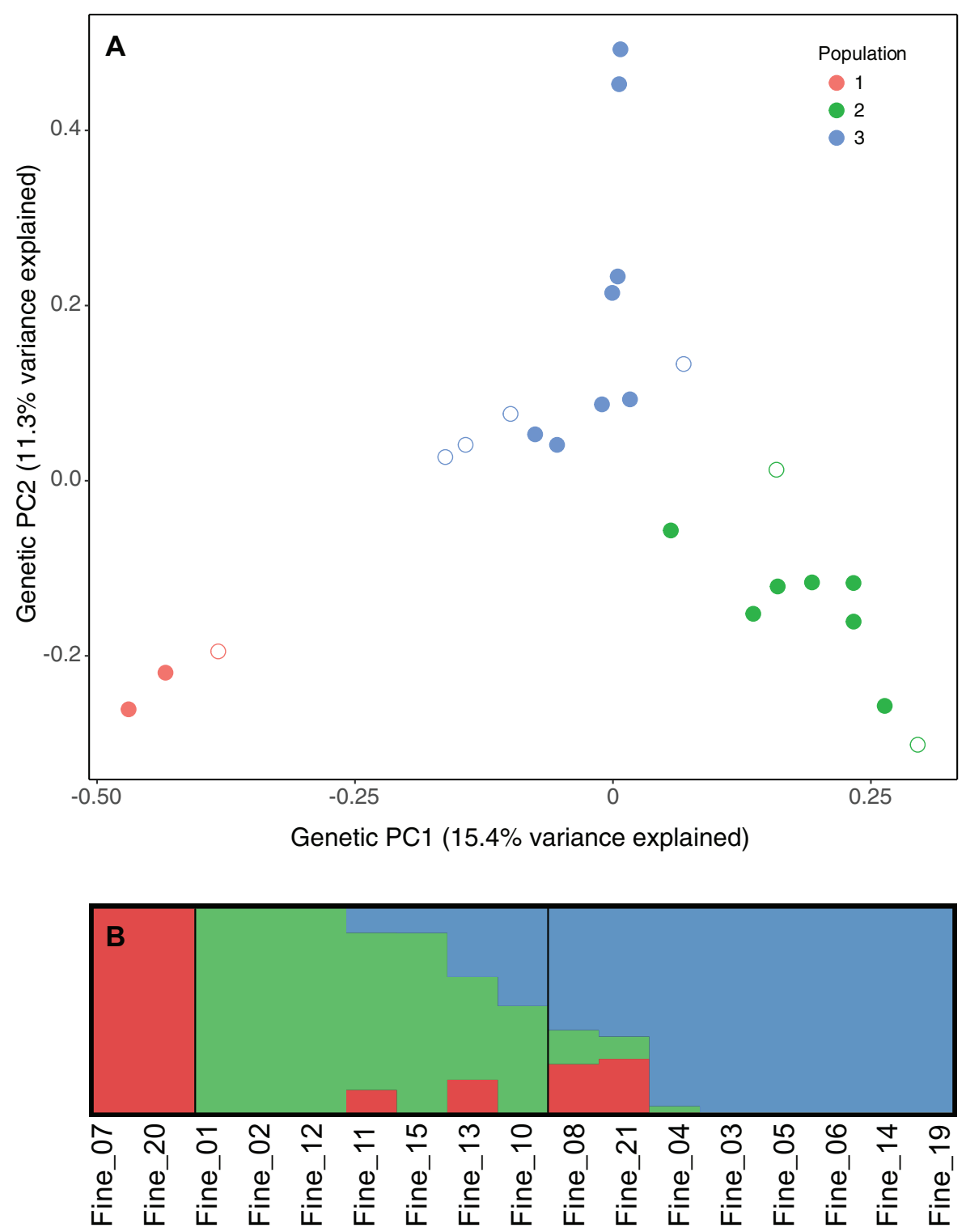

Figure 1 (A) A genetic PCA of all 24 unique fine-textured genotypes, based on data from Goad et al. (2021). The first and second principal components explain $15.4 \%$ and $11.3 \%$ of the variance, respectively. Genotypes are colored based on assignment to genetic population by ADMIXTURE. Open circles indicate genotypes that were not phenotyped in this study. (B) A visualization of population assignment from ADMIXTURE for the subset of

17 genotypes for which we analyzed phenotype data. Vertical black lines divide genotypes into three populations (1, 2, and 3 from left to right). Admixed individuals were assigned to the population for which the membership coefficient was greater than $50 \%$.

$\mathrm{P}<10^{-16}$; Supplementary Figure S4B). These correlations indicate that sample weight may have a small effect on ionome quantification, but that it explains a much smaller proportion of the variance than treatment.

Since the weight of the ionomics sample and the total shoot biomass were sometimes the same (for samples up to $125 \mathrm{mg}$ ), we wanted to determine whether the correlations between sample weight and ion concentration may be caused by actual differences in accumulation between plants of different sizes rather than technical variation in sample weight. We examined the 144 samples within the target sample weight range (60-125 mg) because it included samples where collected biomass equaled sample weight. Within this range, no ions showed significant positive correlations between sample weight and ion concentration; however, concentrations of $\mathrm{P}, \mathrm{Rb}$, and several trace metals (Co, Ni, $\mathrm{Cu}, \mathrm{Zn}$, and $\mathrm{Cd}$ ) showed significant negative correlations between collected biomass and ion concentration after controlling for multiple hypothesis testing $\left(P<2.7 \times 10^{-3}\right.$; Supplementary Table S4). Based on this pattern, larger plants may have lower ion concentrations due to uptake being largely constant, resulting in the same quantity of ions being spread across more biomass and thus less concentrated. These results also suggest that the sample weight effect described above is largely driven by smaller samples (25-60 mg), as the sample weight effect is not significant when they are removed.

\section{Elemental differences within and between genotypes}

Many genotypes were represented by accessions that were collected as cuttings directly from wild plants, so might have retained some residual within-genotype phenotypic variation reflecting environmental heterogeneity between collection locations. We found that only four elements (Co, $\mathrm{Cu}, \mathrm{Ni}$, and $\mathrm{Rb}$ ) varied significantly between accessions within a genotype and that 

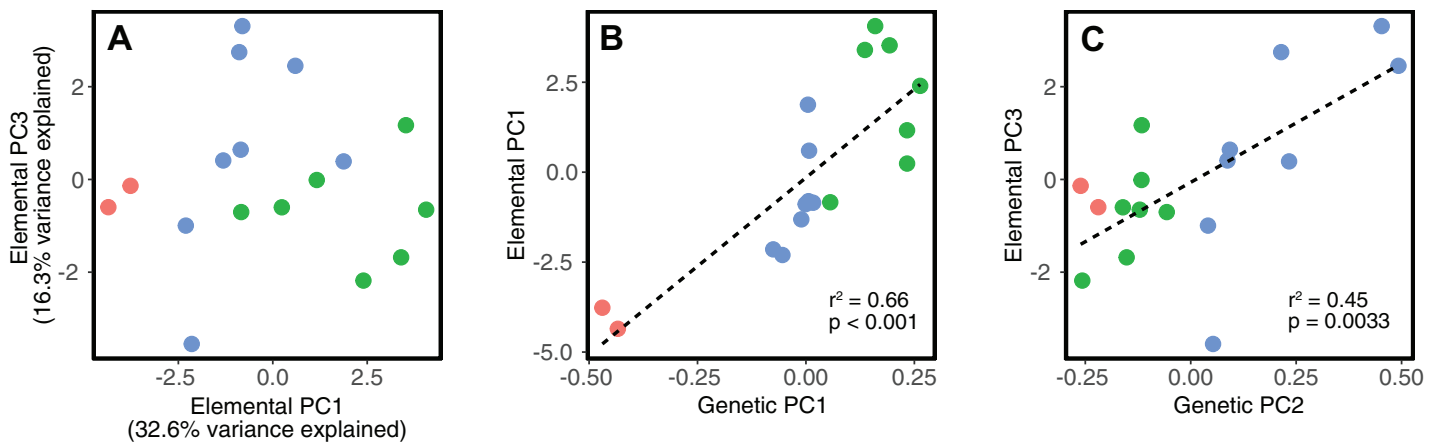

Figure 2 (A) PCA based on the BLUPs calculated for each ion concentration for each genotype. (B) The Spearman correlation between genetic PC1 and elemental PC1. (C) The Spearman correlation between genetic PC2 and elemental PC3. Genotypes are colored based on assignment to the genetic population by ADMIXTURE.

all of the elements except $\mathrm{S}$ and Se had significant betweengenotype differences (Supplementary Table S4). Together these findings suggest that ionomic variation is largely stable but that plastic responses to environmental conditions may not entirely disappear when a plant is moved to a new environment.

\section{Elemental differences between populations}

We quantified the overall elemental profiles of our genotypes by performing a PCA on the BLUPs generated for each of the 19 elements (Figure 2A). The elemental profiles of genotypes clustered based on their population assignment. Furthermore, genetic PC 1 and elemental PC 1 were significantly correlated (Figure 2B), as well as genetic PC 2 and elemental PC 3 (Figure 2C), indicating that more distantly related genotypes have more dissimilar ionomic profiles. Population 1 (comprising two wild accessions from the southeastern US Atlantic Coast) appears to be particularly distinct from the other two populations both genetically and elementally.

\section{Biomass differences between populations}

The three populations also significantly differed in both their overall biomass and the percent change in biomass at increased salt concentrations. During the initial, no salt treatment (2.5 dS/ $\mathrm{m})$, the mean biomass for populations 1, 2, and 3 was 40.8, 120.1, and $94.9 \mathrm{mg}$, respectively $[F(2,14)=4.94, P<0.05$; Supplementary Figure S5]. All three populations showed reduced growth as the salt concentration increased and therefore had a negative percent change in biomass between the no salt and high salt treatments (2.5 and $30 \mathrm{dS} / \mathrm{m}$; Figure 3A). The magnitude of this change can be used as a measure of salt tolerance; notably, it differed significantly between populations $[F(2,14)=16.8, P<0.001]$. Population 2 was least affected by the increased salinity with a $-50.7 \%$ change in biomass. A larger reduction was observed in populations 1 and 3 which had similar percent changes in biomass of $-78.1 \%$ and $-80.8 \%$, respectively. Therefore, population 2 appears to be more salt tolerant than the other two populations.

\section{Correlations between ion concentration and percent change in biomass}

After correction for multiple hypothesis testing, three separate elements showed significant correlations between the ion concentration BLUPs and percent change in biomass of individual genotypes. $\mathrm{K}$ and Fe showed significant positive associations $\left(r^{2}=\right.$ $0.55, P<2.7 \times 10^{-3}$ and $r^{2}=0.54, P<2.7 \times 10^{-3}$ respectively) while Ca showed a significant negative association $\left(r^{2}=0.50, P<2.7 \times\right.$ $10^{-3}$; Figure 4; Supplementary Table S5).
These results suggest that shoot concentrations of $\mathrm{K}$, Fe, and Ca may play some role in salt tolerance. No genotype-bytreatment interactions were significant, indicating that genotypes all adjust ion accumulation in the same way with increased salinity (Supplementary Table S4). Consistent with our findings that different genetic populations also differ in their elemental profiles, these trends follow the expected patterns based on population structure (Figure 3, B-F; Supplementary Figure S5). Genotypes from Population 2 maintain a higher relative biomass in increased salinity and have the highest $\mathrm{K}$ and Fe concentrations as well as the lowest Ca concentrations. Population 1 was on the opposite end of the spectrum for these measurements and Population 3 was intermediate. This pattern indicates that differential accumulation of these three ions between populations may reflect local adaptation for varying salinity levels in the wild.

\section{Discussion \\ Genetic and elemental profile variation within and between genotypes}

Understanding salt tolerance variation in wild species requires consideration of population structure as well as variation in ionomic profiles among genotypes. Our population genetics analyses are the first to explore population structure within fine-textured $P$. vaginatum using genome-wide SNP markers. We recovered both of the genetic populations previously identified in this species (Eudy et al. 2017) (corresponding to our Populations 2 and 3), in addition to a new third population which was unique to our collections from the US Atlantic Coast (Population 1; Figure 1). Our analysis of the elemental profiles of each genotype largely recapitulated the pattern seen in the population structure analysis, with strong correlations between the first PC of both the genetic and elemental datasets as well as between genetic PC 2 and elemental PC 3 (Figure 2). This finding suggests that genotypic differences in elemental accumulation traits have diverged between populations and may be heritable. It is not clear whether this divergence is adaptive since none of the genetic or elemental PCs were significantly associated with variation in the percent change in biomass at increased salinity. It is possible that these population-level differences could reflect adaptations for other stressful conditions that we did not test for, such as drought or metal contamination.

We also found variation within genotypes for shoot accumulation of $\mathrm{Co}, \mathrm{Cu}, \mathrm{Ni}$, and Rb (Supplementary Table S4). All of the accessions used in this experiment were taken as cuttings from wild plants or USDA germplasm rather than grown from seed. Therefore, it is possible that phenotypic plasticity or epigenetic differences between otherwise genetically identical plants from 

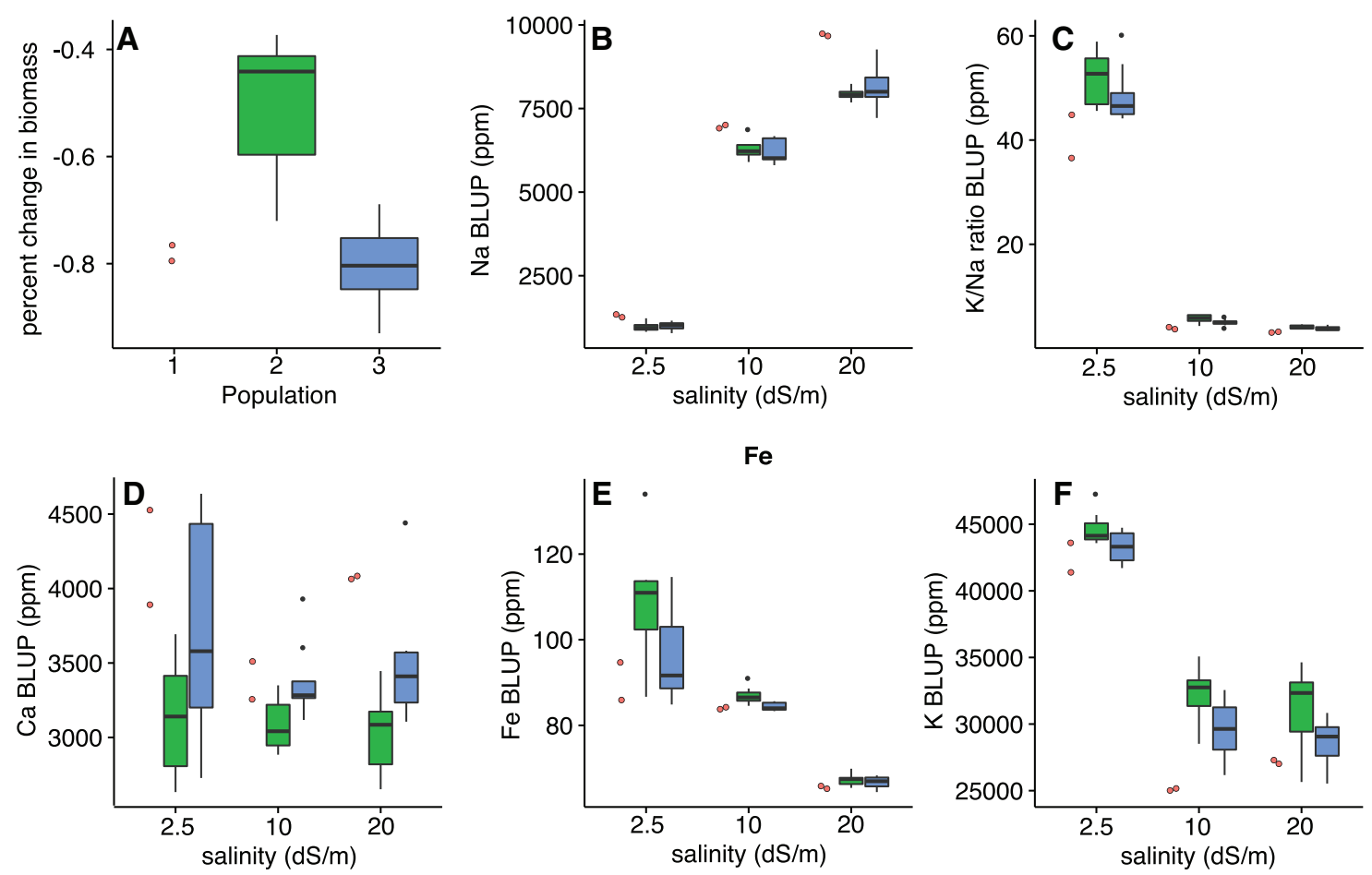

Figure 3 Phenotypic comparison between population 1 (red), population 2 (green), and population 3 (blue). Percent change in biomass at increased salinity (A). Elemental accumulation BLUPs for each treatment: Na (B), K/Na ratio (C), Ca (D), Fe (E), and K (F).
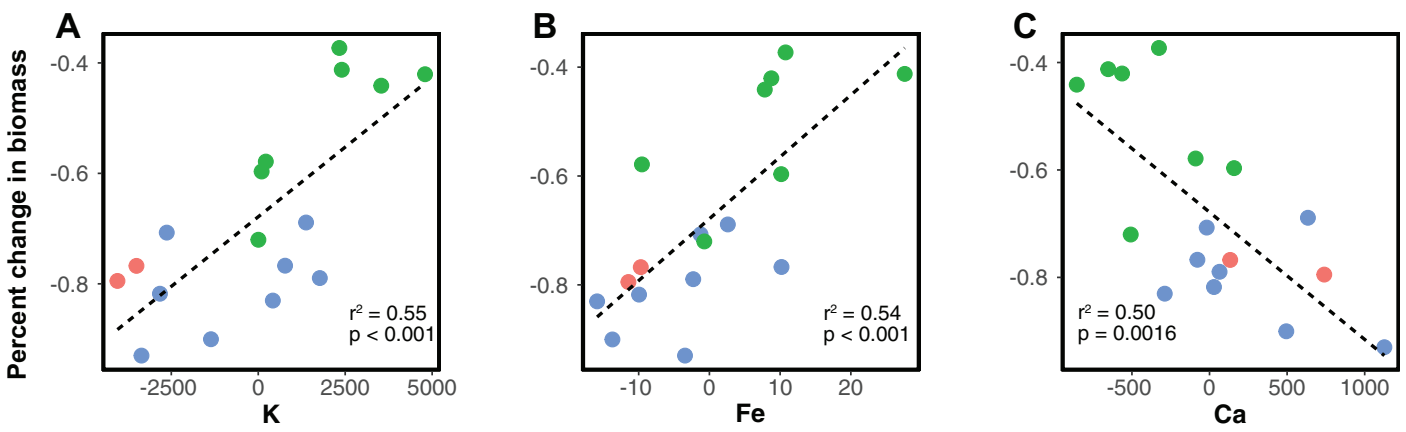

Shoot ion concentration BLUP

Figure 4 Correlations between the percent change in biomass at increased salinity and shoot concentration of $\mathrm{K}(\mathrm{A})$; Fe (B), and Ca (C). Genotypes are colored based on assignment to genetic population by ADMIXTURE.

different locations could still be affecting phenotypes in our common garden experiment (Bossdorf et al. 2008). Paspalum vaginatum often propagates asexually in the wild (Goad et al. 2021) and plasticity in elemental accumulation could allow clones of a single genotype to have increased fitness across a wider variety of environments (Vrijenhoek and Parker 2009). It is also possible that within-genotype variation could be due to rare polymorphisms that arose from somatic mutation within clonal lineages that were not detected in our GBS dataset. Whole-genome sequencing including a focus on epigenetic modification (e.g., bisulfite sequencing) will be required to understand the relative role of somatic mutations and epigenetics in explaining the variation seen within genotypes.

\section{Correlations between elemental accumulation and salt tolerance}

Maintaining tissue ion homeostasis is one of the major challenges faced by plants in saline environments. The hypertonic environment can cause dehydration unless the plant can regulate osmotic pressure by accumulating ions or organic osmolytes. In some cases, $\mathrm{Na}$ is accumulated at a higher rate to maintain this balance (Baxter et al. 2010). This is not an ideal solution, however, as $\mathrm{Na}$ is cytotoxic at high concentration and can compete with the accumulation of other important ions such as $\mathrm{K}$. Therefore, tightly regulating the uptake of $\mathrm{Na}$ is vital for halophyte survival, and variation between genotypes in these mechanisms could be involved in differential salt tolerance. Like earlier studies (Lee et al. 2007; Guo et al. 2016), we found that neither $\mathrm{Na}$ accumulation nor the $\mathrm{K} / \mathrm{Na}$ ratio were associated with salt tolerance, as measured by percent change in biomass, within P. vaginatum (Supplementary Table S5). While Na accumulation was not significant overall, we did observe that the two genotypes from population 1 appeared to accumulate more Na than the other two populations (Figure 3F; Supplementary Figure S5). A more extensive collection of genotypes from population 1 will be required to determine if higher $\mathrm{Na}$ accumulation plays a role in the population's relatively low salt tolerance. 
Significant correlations with salt tolerance were identified for $\mathrm{K}, \mathrm{Fe}$, and $\mathrm{Ca}$. It is thus possible that differential accumulation of these three elements in the shoots could play a role in the variation in tolerance seen within fine-textured P. vaginatum. Below, we discuss the potential biological roles that these three elements could be playing in salt tolerance variation.

\section{Potassium}

$\mathrm{K}$ accumulation plays a key role in salt tolerance in both halophytes and glycophytes (Shabala and Cuin 2008). Due to its similarity to $\mathrm{Na}$ in both size and charge, $\mathrm{K}$ accumulation faces direct competition with $\mathrm{Na}$. Compared to $\mathrm{Na}$, however, $\mathrm{K}$ is more vital for plant function and less toxic (Shabala and Cuin 2008). Halophytic species tend to accumulate more $\mathrm{K}$ under saline conditions than glycophytes (Ghars et al. 2008; Edelist et al. 2009), and intraspecific variation in $\mathrm{K}$ accumulation is associated with increased salt tolerance in many glycophytes (Chen et al. 2005; Chakraborty et al. 2016). The role of $\mathrm{K}$ accumulation in salt tolerance variation within halophytic species is less well understood. Fine-textured $P$. vaginatum has been shown to have increased $\mathrm{K}$ accumulation compared to the less tolerant P. distichum and coarse-textured P. vaginatum accessions (Spiekerman and Devos 2020). This difference may be due to the increased size of adaxial leaf papillae in fine-textured plants; these structures sequester $\mathrm{Na}$ and $\mathrm{K}$, which could allow for greater ion accumulation (Spiekerman and Devos 2020). The role that variation in $\mathrm{K}$ accumulation plays within fine-textured accessions is less well characterized. Previous studies have identified a difference in shoot $\mathrm{K}$ accumulation between the most and least tolerant accessions; however, there was no correlation between $\mathrm{K}$ and salt tolerance when comparing all of the sampled accessions (Lee et al. 2007; Guo et al. 2016; Wu et al. 2020). Our results are thus the first to show that $\mathrm{K}$ accumulation is associated with increased tolerance across multiple fine-textured varieties. It is possible that, as with leaf morphology differences between coarse- and fine-textured morphotypes, variation among fine-textured plants in the size and function of the adaxial leaf papillae could explain this trend. If such differentiation exists our results suggest that it is the accumulation of $\mathrm{K}$ rather than $\mathrm{Na}$ that drives the variation as neither $\mathrm{Na}$ nor the $\mathrm{K} / \mathrm{Na}$ ratio were associated with tolerance. Since we measured accumulation in the entire shoot, we could not differentiate between the $\mathrm{K}$ content of the adaxial leaf papilla and other structures. Therefore, a more thorough survey of the adaxial papillae in a large sample of fine-textured genotypes would be useful to test this hypothesis on their role in $\mathrm{K}$ and $\mathrm{Na}$ accumulation.

\section{Iron}

The accumulation of Fe was also associated with increased tolerance in our experiment. This finding is consistent with observations in glycophytes that increases in environmental Fe concentration, and thus accumulation, can alleviate the negative impacts of high salinity (Ghasemi et al. 2014). Fe plays an important role in photosynthesis and homeostasis of reactive oxygen species. In plant tissues, free Fe is sequestered in a nontoxic form by storage proteins called ferritins. Halophytes exposed to salinity have been shown to have increased expression of ferritin genes and to exhibit higher Fe concentrations, particularly in the chloroplast where ferritin may improve the efficiency of photosynthesis during salt stress (Paramonova et al. 2004; Jithesh et al. 2006). Within P. vaginatum, the Fe transporter protein IRT1 has been shown to be differentially expressed in high salinity environments, and transgenic yeast containing a copy of the IRT1 gene from P. vaginatum showed increased salt tolerance (Chen et al. 2016). Together these findings suggest that Fe accumulation, ferritin production and IRT1 may have some influence in determining the level of salt tolerance of a P. vaginatum genotype.

\section{Calcium}

Calcium plays an important role in plant physiology, particularly as a structural component of the cell wall and membranes and as a secondary messenger in a wide range of signaling networks (Thor 2019). It is also involved in plant salt tolerance as part of the SOS pathway which regulates $\mathrm{K}$ and $\mathrm{Na}$ homeostasis (Mahajan et al. 2008). In multiple species, supplemental Ca has been shown to ameliorate the negative effects of salinity stress (Carvajal et al. 2000; Sun et al. 2009; Yousuf et al. 2015; Larbi et al. 2020). Therefore, our finding that increased shoot Ca concentrations are associated with reduced salt tolerance in P. vaginatum was surprising. It is possible that the shoot $\mathrm{Ca}$ concentration may not directly affect salt tolerance, but instead reflects differences in transpiration and water conservation between genotypes.

Unlike many solutes, long-range transport of Ca to the shoots primarily follows an apoplastic pathway and is therefore strongly tied to the rate of transpiration rather than membrane-bound calcium transporters which are primarily involved in signaling on a more local scale (White 2001). Within P. vaginatum some genotypes have been shown to more quickly reduce transpiration in response to drying soil as a water conservation strategy (Johnson et al. 2009). It is thus possible that in our experiment the drying of the growth medium between flooding events could have triggered these responses. If this is the case, the plants that maintained normal transpiration rates for a longer period of time could have accumulated more shoot $\mathrm{Ca}$, but been more susceptible to osmotic stress at higher salinity concentration due to increased water loss. A more focused study involving explicit measures of transpiration rate will be required to better understand the interactions between salt tolerance, transpiration, and Ca accumulation in P. vaginatum.

\section{Conclusions}

Intraspecific variation in salt tolerance within halophytes remains poorly understood. Here we showed that genetic populations of P. vaginatum differ in their elemental accumulation traits and that salt tolerance is associated with the accumulation of specific ions. These finding suggest that a population-level approach to studying halophytes may be a valuable, and so far, untapped, source of knowledge regarding salt tolerance genes and mechanisms for breeding more tolerant crop varieties. However, our results are correlational in nature. Therefore, future work investigating the relationship between salt tolerance and the root ionome, transpiration rate, and differentially expressed ion transporters will be necessary to shed further light on the mechanisms controlling salt tolerance in P. vaginatum and other halophytes. Nonetheless, breeders interested in improving seashore paspalum for use as a turfgrass may be able to select for optimal K, Fe, and $\mathrm{Ca}$ accumulation traits to improve salt tolerance. Other elements which showed differential accumulation between genotypes and populations may also play a role in adaptation to other stressors (e.g., drought, metal contamination, poor soil quality) and could also be of interest for turf breeding. 


\section{Data availability}

Demultiplex and trimmed reads from Goad et al. (2021) are available at the NCBI SRA under project ID PRJNA669382. The SNP datasets from Goad et al. (2021) are available on Dryad at https:// doi.org/10.5061/dryad.vt4b8gtqh. Ionomics and biomass data are included in Supplementary Table S3. All scripts used for data analysis and for controlling the irrigation system are available at https://github.com/david-goad/paspalum_ionomics.

Supplementary material is available at figshare: https://doi.org/ $10.25387 / g 3.15018981$

\section{Acknowledgments}

The authors thank Kevin Riley, Sally Fabbri, and the rest of the Danforth Center greenhouse staff for assistance with plant care and irrigation system development; Marshall Wedger and Taylor Aubuchon-Elder for assisting with tissue collection; Melissa Jurkowski for running the ionomics pipeline; and Greg Ziegler for providing barcoded sample labels and data analysis advice.

\section{Funding}

Funding for this project was provided by the US Golf Association (project 2016-35-605). D.M.G. was supported by the William H. Danforth Plant Science Fellowship and the Donald Danforth Plant Science Center.

\section{Conflicts of interest}

The authors declare that there is no conflict of interest.

\section{Literature cited}

Alexander DH, Novembre J, Lange K. 2009. Fast model-based estimation of ancestry in unrelated individuals. Genome Res. 19: 1655-1664.

Ali A, Yun D-J. 2017. Salt stress tolerance; what do we learn from halophytes? J Plant Biol. 60:431-439.

Baxter I, Brazelton JN, Yu D, Huang YS, Lahner B, et al. 2010. A coastal cline in sodium accumulation in Arabidopsis thaliana is driven by natural variation of the sodium transporter AtHKT1;1. PLOS Genet. 6:e1001193.

Baxter IR, Vitek O, Lahner B, Muthukumar B, Borghi M, et al. 2008. The leaf ionome as a multivariable system to detect a plant's physiological status. Proc Natl Acad Sci U S A. 105:12081-12086.

Bossdorf O, Richards CL, Pigliucci M. 2008. Epigenetics for ecologists. Ecol Lett. 11:106-115.

Carvajal M, Cerdá A, Martínez V. 2000. Does calcium ameliorate the negative effect of $\mathrm{NaCl}$ on melon root water transport by regulating aquaporin activity? New Phytol. 145:439-447.

Chakraborty K, Bose J, Shabala L, Shabala S. 2016. Difference in root $\mathrm{K}+$ retention ability and reduced sensitivity of $\mathrm{K}+$-permeable channels to reactive oxygen species confer differential salt tolerance in three Brassica species. J Exp Bot. 67:4611-4625.

Cheeseman JM. 2013. The integration of activity in saline environments: problems and perspectives. Funct Plant Biol. 40:759-774.

Cheeseman JM. 2015. The evolution of halophytes, glycophytes and crops, and its implications for food security under saline conditions. New Phytol. 206:557-570.

Chen J, Yan J, Qian Y, Jiang Y, Zhang T, et al. 2009. Growth responses and ion regulation of four warm season turfgrasses to long-term salinity stress. Sci Hortic. 122:620-625.
Chen Y, Chen C, Tan Z, Liu J, Zhuang L, et al. 2016. Functional identification and characterization of genes cloned from halophyte seashore paspalum conferring salinity and cadmium tolerance. Front Plant Sci. 7:102.

Chen Z, Newman I, Zhou M, Mendham N, Zhang G, et al. 2005. Screening plants for salt tolerance by measuring $\mathrm{K}+$ flux: a case study for barley. Plant Cell Environ. 28:1230-1246.

Davies L, Gather U. 1993. The identification of multiple outliers. J Am Stat Assoc. 88:782-792.

Edelist C, Raffoux X, Falque M, Dillmann C, Sicard D, et al. 2009. Differential expression of candidate salt-tolerance genes in the halophyte Helianthus paradoxus and its glycophyte progenitors $H$. annuus and H. petiolaris (Asteraceae). Am J Bot. 96:1830-1838.

Eudy D, Bahri BA, Harrison ML, Raymer P, Devos KM. 2017. Ploidy level and genetic diversity in the genus paspalum, group Disticha. Crop Sci. 57:3319-3332.

Ghars MA, Parre E, Debez A, Bordenave M, Richard L, et al. 2008. Comparative salt tolerance analysis between Arabidopsis thaliana and Thellungiella halophila, with special emphasis on $\mathrm{K}+/ \mathrm{Na}+$ selectivity and proline accumulation. J Plant Physiol. 165:588-599.

Ghasemi S, Khoshgoftarmanesh AH, Afyuni M, Hadadzadeh H. 2014. Iron(II)-amino acid chelates alleviate salt-stress induced oxidative damages on tomato grown in nutrient solution culture. Sci Hortic. 165:91-98.

Goad DM, Baxter I, Kellogg EA, Olsen KM. 2021. Hybridization, polyploidy and clonality influence geographic patterns of diversity and salt tolerance in the model halophyte seashore paspalum (Paspalum vaginatum). Mol Ecol. 30:148-161.

Guo H, Wang Y, Li D, Chen J, Zong J, et al. 2016. Growth response and ion regulation of seashore paspalum accessions to increasing salinity. Environ Exp Bot. 131:137-145.

Jithesh MN, Prashanth SR, Sivaprakash KR, Parida A. 2006. Monitoring expression profiles of antioxidant genes to salinity, iron, oxidative, light and hyperosmotic stresses in the highly salt tolerant grey mangrove, Avicennia marina (Forsk.) Vierh. by mRNA analysis. Plant Cell Rep. 25:865-876.

Johnson GL, Sinclair TR, Kenworthy K. 2009. Transpiration and normalized difference vegetation index response of seashore paspalum to soil drying. HortScience. 44:2046-2048.

Kumari A, Das P, Parida AK, Agarwal PK. 2015. Proteomics, metabolomics, and ionomics perspectives of salinity tolerance in halophytes. Front Plant Sci. 6:537.

Larbi A, Kchaou H, Gaaliche B, Gargouri K, Boulal H, et al. 2020. Supplementary potassium and calcium improves salt tolerance in olive plants. Sci Hortic. 260:108912.

Lee G, Carrow RN, Duncan RR. 2004a. Photosynthetic responses to salinity stress of halophytic seashore paspalum ecotypes. Plant Sci. 166:1417-1425.

Lee G, Carrow RN, Duncan RR. 2005. Growth and water relation responses to salinity stress in halophytic seashore paspalum ecotypes. Sci Hortic. 104:221-236.

Lee G, Carrow RN, Duncan RR, Eiteman MA, Rieger MW. 2008. Synthesis of organic osmolytes and salt tolerance mechanisms in Paspalum vaginatum. Environ Exp Bot. 63:19-27.

Lee G, Duncan RR, Carrow RN. 2007. Nutrient uptake responses and inorganic ion contribution to solute potential under salinity stress in halophytic seashore paspalums. Crop Sci. 47:2504-2512.

Lee G, Duncan RR, Carrow RN. 2004b. Salinity tolerance of seashore paspalum ecotypes: shoot growth responses and criteria. HortScience. 39:1138-1142.

Mahajan S, Pandey GK, Tuteja N. 2008. Calcium- and salt-stress signaling in plants: shedding light on SOS pathway. Arch Biochem Biophys. 471:146-158. 
Paramonova NV, Shevyakova NI, Kuznetsov VV. 2004. Ultrastructure of chloroplasts and their storage inclusions in the primary leaves of Mesembryanthemum crystallinum affected by putrescine and $\mathrm{NaCl}$. Russ J Plant Physiol. 51:86-96.

Parida AK, Das AB. 2005. Salt tolerance and salinity effects on plants: a review. Ecotoxicol Environ Saf. 60:324-349.

Purcell S, Neale B, Todd-Brown K, Thomas L, Ferreira MAR, et al. 2007. PLINK: a tool set for whole-genome association and population-based linkage analyses. Am J Hum Genet. 81: 559-575.

Sanchez DH, Pieckenstain FL, Escaray F, Erban A, Kraemer U, et al. 2011. Comparative ionomics and metabolomics in extremophile and glycophytic Lotus species under salt stress challenge the metabolic pre-adaptation hypothesis. Plant Cell Environ. 34:605-617.

Saslis-Lagoudakis CH, Moray C, Bromham L. 2014. Evolution of Salt Tolerance in Angiosperms: A Phylogenetic Approach. In: Rajakaruna N, Boyd RS, Harris TB, editors. Saslis-Lagoudakis CH, Moray C, Bromham L. Evolution of salt tolerance in angiosperms: a phylogenetic approach. Plant ecology and evolution in harsh environments. New York: Hauppage. p. 77-95.

Shabala S, Cuin TA. 2008. Potassium transport and plant salt tolerance. Physiol Plant. 133:651-669.

Shahid SA, Zaman M, Heng L. 2018. Soil salinity: historical perspectives and a world overview of the problem. In: M Zaman, SA Shahid, L Heng, editors. Guideline for Salinity Assessment, Mitigation and Adaptation Using Nuclear and Related Techniques. Cham: Springer International Publishing. p. 43-53.

Spiekerman JJ, Devos KM. 2020. The halophyte seashore paspalum uses adaxial leaf papillae for sodium sequestration. Plant Physiol. 184:2107-2119.
Sun J, Dai S, Wang R, Chen S, Li N, et al. 2009. Calcium mediates root $\mathrm{K}+/ \mathrm{Na}+$ homeostasis in poplar species differing in salt tolerance. Tree Physiol. 29:1175-1186.

Szabolcs I. 1989. Salt-Affected Soils. Boca Raton, FL: CRC Press.

Thor K. 2019. Calcium—nutrient and messenger. Front Plant Sci. 10: 440.

Vrijenhoek RC, Parker ED. 2009. Geographical parthenogenesis: general purpose genotypes and frozen niche variation. In: I Schön, K Martens, P Dijk, editors. In Lost Sex: The Evolutionary Biology of Parthenogenesis. Dordrecht: Springer Netherlands. p. 99-131.

White PJ. 2001. The pathways of calcium movement to the xylem. J Exp Bot. 52:891-899.

Wu P, Cogill S, Qiu Y, Li Z, Zhou M, et al. 2020. Comparative transcriptome profiling provides insights into plant salt tolerance in seashore paspalum (Paspalum vaginatum). BMC Genomics. 21: 131.

Yermiyahu U, Nir S, Ben-Hayyim G, Kafkafi U. 1994. Quantitative competition of calcium with sodium or magnesium for sorption sites on plasma membrane vesicles of melon (Cucumis melo L.) root cells. J Membr Biol. 138:55-63.

Yousuf PY, Ahmad Hemant, A, Ganie AH, Aref IM, et al. 2015. Potassium and calcium application ameliorates growth and oxidative homeostasis in salt-stressed Indian mustard (Brassica juncea) plants. Pak J Bot. 47:1629-1639.

Ziegler G, Terauchi A, Becker A, Armstrong P, Hudson K, et al. 2013. Ionomic screening of field-grown soybean identifies mutants with altered seed elemental composition. The Plant Genome. 6: plantgenome2012.07.0012.

Communicating editor P. Inguarsson 